\title{
Plan de manejo de gobernanza ambiental para la reubicación de epifitas ante la ejecución de obras viales: vereda "el páramo", pamplonita, Colombia
}

\author{
Environmental governance management plan for the relocation of epiphytes before the execution of road works: Vereda \\ “El Páramo”, Pamplonita, Colombia
}

\author{
Carol Rojas ${ }^{a}$; Yolanda Hernández ${ }^{a}$ \\ ${ }^{a}$ Universidad Distrital Francisco José de Caldas, Colombia
}

\section{Resumen}

Para solventar el impacto ambiental sobre la flora epífita por obras viales, se realiza rescate y reubicación de estas plantas para conservar su acervo genético y mantener los servicios ecosistémicos que ofrecen, pero la inclusión de la comunidad en estos proyectos de preservación ex situ es limitada, teniendo una gobernanza ambiental baja. El objetivo de esta investigación fue identificar los actores sociales implicados en el proceso de reubicación de flora epífita afectada por la construcción de la Doble vía Cúcuta-Pamplona unidad funcional 1 por medio de un Mapeo de actores clave (MAC), conocer sus percepciones sobre la ejecución de compensación e identificar su participación aplicando entrevistas semiestructuradas, para formular un Plan de Manejo basado en los principios de la Gobernanza ambiental y fortalecer los resultados de conservación. Este modelo fue socializado y discutido en un Grupo Focal con los líderes de los actores clave implicados, donde se aprobó su idoneidad en proyectos que vinculen el rescate y reubicación de flora epífita. La metodología aplicada en esta investigación tuvo enfoque cualitativo. El acercamiento con los actores permitió un enriquecimiento de saberes de interés investigativo y académico, el cual fue valorado para profundizar en el comportamiento hacia la conservación de la naturaleza, credibilidad en el proceso de conservación y apropiación del territorio.

Palabras clave: Gobernanza ambiental; Epífitas; Reubicación; Comunidad.

\begin{abstract}
To solve the environmental impact on the epiphytic flora by road works, these plants are rescued and relocated to conserve their genetic heritage and maintain the ecosystem services they offer, but the inclusion of the community in these ex-situ preservation projects is limited. having low environmental governance. The objective of this research was to identify the social actors involved in the relocation process of epiphytic flora affected by the construction of the Cúcuta-Pamplona Double Road, functional unit 1 through a Mapping of key actors (MAC), to know their perceptions about the execution of compensation and identify their participation applying semi-structured interviews, to formulate a Management Plan based on the principles of Environmental Governance and strengthen conservation results. This model was socialized and discussed in a Focus Group with the leaders of the key stakeholders involved, where its suitability in projects that link the rescue and relocation of epiphytic flora was approved. The methodology applied in this research had a qualitative approach. The approach with the actors allowed an enrichment of knowledge of investigative and academic interest, which was valued to deepen the behavior towards the conservation of nature, credibility in the conservation process and appropriation of the territory.
\end{abstract}

Keywords: Environmental governance; Epiphytes; Relocation; Community.

\section{Introducción}

La sobreexplotación de los servicios ecosistémicos impulsados por el sistema extractivista del capitalismo, y en general del modelo económico actual, ha ocasionado problemáticas ambientales a nivel global; situación que ha hecho necesario el surgimiento de nuevos conceptos desde la academia que frenen estos daños y generen un cambio, ya que al sobreexplotar los servicios ecosistémicos de un lugar se genera pérdida y degradación de los ecosistemas, siendo una amenaza inminente para el suministro y flujo continuo de estos servicios de los cuales dependen las generaciones de todos los seres vivos. Es así como han surgido los conceptos como sustentabilidad y gobernanza, el primero de ellos creado para generar el cambio y el segundo como herramienta para lograrlo. Naranjo et al [18], definen la gobernanza como un proceso donde prima la coordinación de actores sociales e instituciones con el fin de alcanzar 
objetivos particulares, discutidos y definidos colectivamente para encontrar la solución adecuada; donde el Estado también se ve transformado e impactado por la sociedad y la sociedad es transformada por el Estado y otros actores.

Dentro de las actividades antrópicas que intervienen los ecosistemas están los proyectos viales que si bien permiten un crecimiento económico, generan impacto ambiental, al intervenir bosques maduros que poseen especies de flora con crecimiento epífito (Plantas que crecen fuera del suelo). Para Larrota [14], " este tipo de plantas poseen un papel fundamental dentro de los ecosistemas tropicales, su valor ecológico radica en que aportan una gran cantidad de biomasa, regulan el flujo hídrico del bosque, fijan nutrientes con sus raíces aéreas que se encuentran directamente en la atmósfera y los incorporan al ciclo trófico del bosque" (p. 2); además, estas plantas ofrecen espacio para el crecimiento de diversos artrópodos y oferta de agua durante la época seca; por su crecimiento vertical sobre otros árboles le otorgan al ecosistema una mayor diversidad, llegando a estimar el 50\% y son bioindicadores de conservación [9] [13].

En busca de proteger esta diversidad el gobierno de Colombia, por medio del entonces Instituto Nacional de los Recursos Naturales Renovables y del Ambiente (INDERENA), estableció la Resolución 0213 de 1977, legislación ambiental para proteger la flora epífita otorgándoles veda, entendiéndose esta categoría como, "una restricción y regulación del uso o aprovechamiento de determinadas especies, grupos taxonómicos o productos de la flora en el territorio a nivel regional o nacional, por un tiempo parcial o temporal" [23, p. 13], para el caso de la flora en veda vascular se destacan las familias de orquídeas (Orchidaceae) y bromelias (Bromeliaceae), las cuales se destacan por su gran diversidad y susceptibilidad ante los cambios estructurales del ecosistema; además, algunas de estas especies se encuentran según la Unión Internacional para la Conservación de la Naturaleza [19] con algún grado de amenaza por las actividades antrópicas.

Desde el 2013, en Colombia se viene desarrollando mejoras en las conexiones viales por medio de la construcción de $8.000 \mathrm{~km}$ de vías (Carreteras 4G), con el fin de obtener una ventaja competitiva en el comercio nacional e internacional al mejorar la conectividad del país [26]; pero que simultáneamente ocasiona un impacto ambiental en los ecosistemas intervenidos, incluida las epífitas, y que requiere implementar los principios de la gobernanza ambiental: eficacia, equidad, transparencia y legitimación, donde la democracia este entre los entes públicos o privados con la población local y sus procesos administrativos sean legítimos [3] Se formuló un Plan de manejo con el fin de observar una conservación más efectiva; según Indij y Schreider [11], esta herramienta articula y organiza las pautas para que se cumplan las estrategias, decisiones, acciones y resultados, buscados en los espacios participativos, donde su objetivo principal es resaltar los aspectos claves en cada fase con el fin de lograr las metas establecidas.

El municipio de Pamplonita está involucrado en el proyecto vial Doble calzada Cúcuta-Pamplona Unidad Funcional 1 y se conformó en la vereda El Páramo un epifitario (zona donde de reubican las epífitas rescatadas), por ello, para la formulación del Plan de manejo fueron tenidas en cuenta las percepciones de los actores sociales de la vereda "El Páramo".

\section{Método}

La investigación se realizó durante marzo y noviembre del 2020. Inicialmente, se revisó la Resolución 2123 del 09 de noviembre del 2018 y del Auto 347 del 27 de agosto del 2019 para realizar un mapeo de actores claves (MAC), que es un elemento de partida para conocer la intervención social que se sostiene a nivel local o regional, representa la intervención real de los actores y su inclusión en proyectos ambientales. El MAC permite dar cuenta de las relaciones sociales entre las organizaciones, instituciones, entidades y/o individuos, facilitando identificar el interés de los mismos, con respecto al contexto ambiental del municipio, de la región, y dentro del territorio [25]. Para hallar los posibles actores indirectos se aplicó el método No probabilístico en Cadena o de Bola de Nieve a los actores principales [10]

Seguidamente, se realizaron entrevistas semiestructuradas a los líderes de los actores identificados para obtener una triangulación en la información, y conocer su percepción sobre el proceso de reubicación de las epífitas. Se hizo uso del Software Atlas Ti v.8.5 para analizar las entrevistas y categorizar las respuestas; este software es una herramienta de análisis de datos cualitativos cuyo objetivo es facilitar el manejo de grandes volúmenes de datos textuales [24] [27]. A partir de estas percepciones y también las pautas dadas por Malambo [16] y Pérez [20] para impulsar la gobernanza, se formuló el plan de manejo donde se promueva la inclusión de la ciencia en la práctica social y la academia acompañe los procesos de toma de decisiones, como es la planificación del territorio y su cuidado ambiental.

Finalmente, se conformó un grupo focal con los actores implicados para socializar y discutir el plan de manejo formulado; esta es una técnica importante de investigación cualitativa cuyo uso se ha incrementado considerablemente en todos los campos de las ciencias humanas [2]. El encuentro tuvo dos fases, la primera consistió en presentación de cada participante y recorrido por el epífitario para conocer el proceso de reubicación y monitoreo de las plantas epífitas; y la segunda fase en discutir el plan de manejo planteado y definir como entre los actores se puede mejorar la gobernanza ambiental en la vereda "El Páramo" y su papel en la conservación. Los métodos participativos agregan experiencias locales vividas ante un cambio ambiental, que son necesarias si se desea una gestión de los recursos naturales que en concordancia con la comunidad implicada [12] 


\subsection{Localización}

El Municipio de Pamplonita se localiza en la Región SurOccidental del Departamento Norte de Santander. La vereda El Páramo, está ubicada a una hora del casco urbano y su acceso vial es por carretera destapada con ciertos tramos de placa huella. No cuenta con transporte público veredal y las familias allí ubicadas se trasladan por medio de caballos, motos o a pie. Se conforma por $806 \mathrm{Ha}$, donde 51 hectáreas son destinadas como Reserva Forestal [5].

Allí viven alrededor de 65 familias, donde su principal actividad economía es la agricultura, donde se destacan los cultivos de papa, arracacha, frijol, cebolla junca, alverja, lulo, durazno, ciruela, fresa, tomate de árbol, mora, y a baja escala la producción de bovinos y porcinos. En el sitio llamado "La Hoyada" se conformó el epífitario "La Manuela" distribuido en 1,36 Ha y con 998 individuos de la familia Bromeliaceae y Orchidaceae, reubicados en suelo y forófitos. El área de estudio presenta una zona de vida según Holdridge, de Bosque seco montano bajo, ubicado en el gran bioma de Bosque Húmedo Tropical [6].

\section{Resultados}

Los actores directos en el proceso de rescate, traslado, reubicación y monitoreos de la flora epífita a conservar, fueron: el Ministerio de Ambiente y Desarrollo Sostenible por medio del departamento de Bosques, biodiversidad y Servicios Ecosistémicos, la concesión Unión Río Pamplonita, Corporación Autónoma de la Frontera Nororiental (CORPONOR) y la Alcaldía de Pamplonita. Por medio de las entrevistas semiestructuradas a los actores regionales y aplicando el método en cadena o bola de nieve, se evidenciaron los actores indirectos, con incidencia baja, pero necesaria el éxito del proceso; y fueron: la Junta de acción comunal de la vereda El Páramo, la Universidad de Pamplona, la institución educativa Nuestra Señora del Pilar sede de la vereda El Páramo, y los agricultores y comunidad de la vereda El Páramo, donde se resalta a la comunidad como los principales actores encargados de conservar la zona donde se realizó la reubicación de las epifitas (ver Fig. 1).

La entrevista semiestructurada permitió identificar como se relacionan los actores sociales con la biodiversidad y las medidas que están dispuestas a ejecutar para lograr su conservación. Durante el proceso se dio el espacio para aclarar dudas sobre el proceso de rescate y reubicación y conceptos técnicos. Ante la necesidad de comprender la importancia que los líderes le otorgan a la vegetación, se encontró unanimidad en su gran valor para la sociedad y la naturaleza misma. Todos afirman la necesidad de protegerla y reconocen que es el soporte para la existencia del hombre (ver Fig. 2).

Dentro de la vegetación encontramos a las plantas epífitas y los actores implicados tampoco desconocen su importancia, relacionándolas con el agua, la permanencia de los pequeños vertebrados e invertebrados, el aporte a la humedad del bosque y su necesidad de conservación (ver Fig. 3).

Al conocer las relaciones entre los actores clave ante la reubicación de flora epífita en la vereda, se evidenció que es poca la interacción que manejan entre los actores clave directos con los indirectos, desconociendo proyectos y medios para solucionar las problemáticas ambientales de la región, siendo baja la gobernanza ambiental en para este tipo de proyectos de conservación.

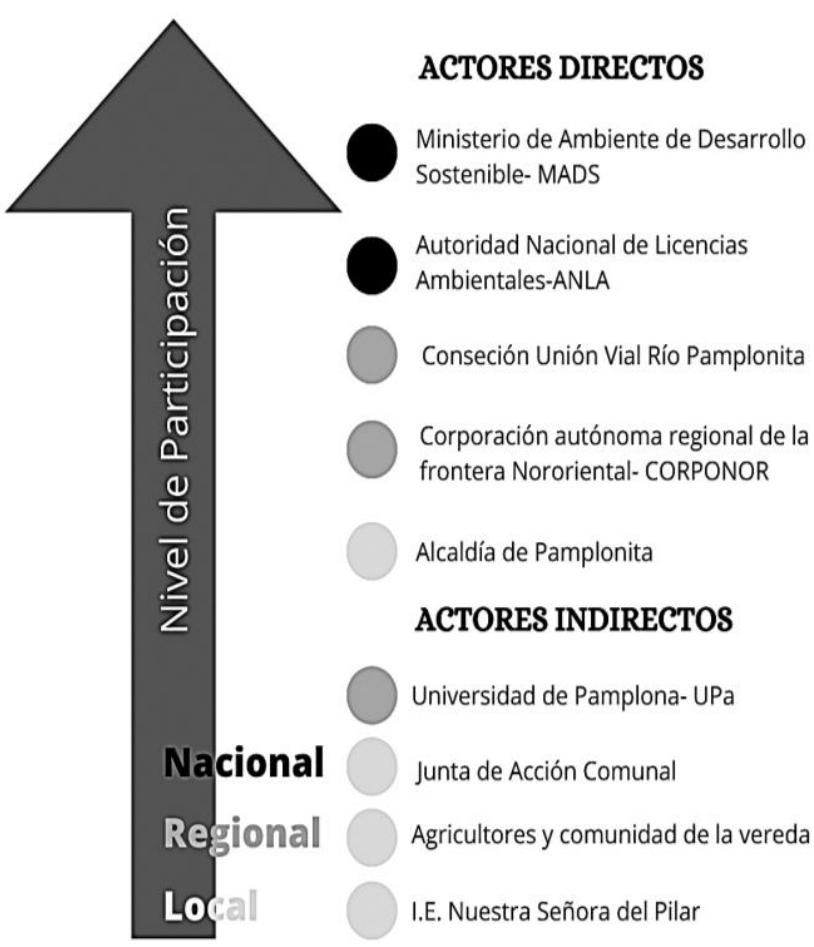

Figura 1. Esquema de actores implicados en el rescate y reubicación de flora epífita en el municipio de Pamplonita

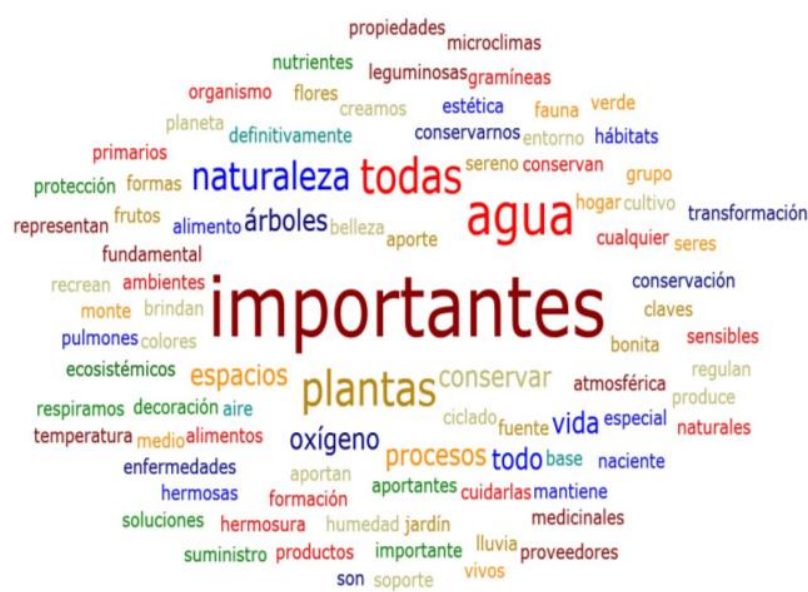

Figura 2. Nube de palabras con percepción de actores de la vereda El Páramo con relación a la vegetación. 


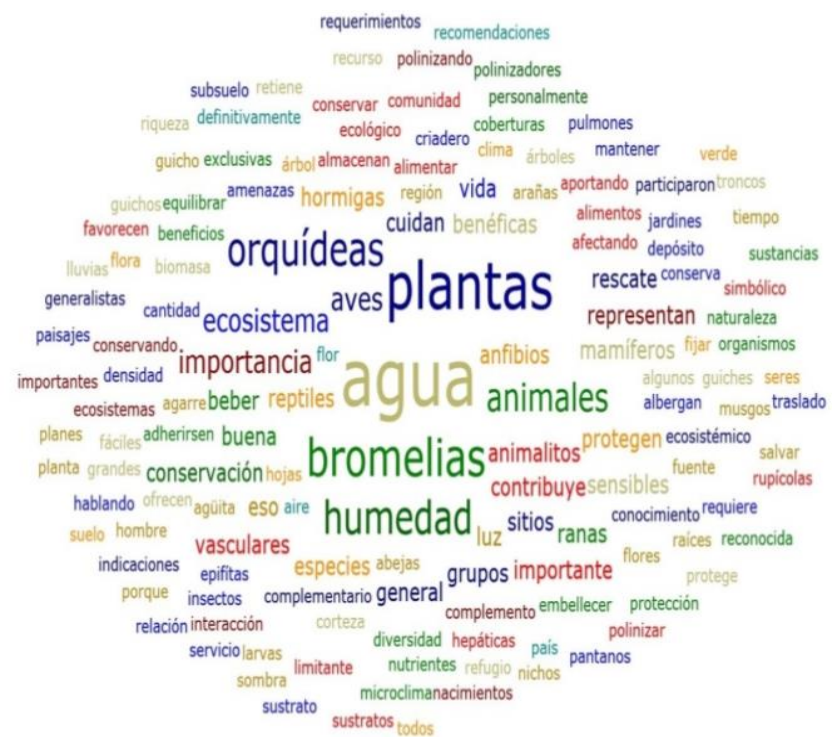

Figura 3. Nube de palabras con percepción de actores sociales de la vereda El Páramo con relación a la flora epífita.

A continuación, se muestran algunas percepciones mencionadas (no se hace mención de los nombres de los entrevistados debido a confidencialidad de datos):

“El rescate es más simbólico para mí".

"La interacción que se tiene en el epifitario es a nivel informativo".

"Tengo poco conocimiento hasta ahora de esto, no estoy muy seguro, dijeron que iban a sembrar, pero no sé si ya se hará eso, porque hace tiempo no se hace reunión".

"Han subido al cerro, pero como es retirado donde yo vivo uno no sabe que hacen por allá."

"La gente le pregunta a uno que hacen esa gente allá, porque estaban diciendo que estaban llevando animales bravos por allá, que taban llevando osos, culebras. Yo les dije que no sé.”

"Sinceramente se había tomado al inicio con muy poco interés, pero se ha cambiado de visión".

"Falta interacción, nos falta trabajo en equipo, con la corporación y organizaciones municipales, que lamentablemente son en ocasiones funcionan solo con asuntos politiqueros".

Durante las entrevistas se tuvo el espacio para señalar el fin de conformar los epifitarios, sus monitoreos e importancia para el ecosistema y para la vereda El Páramo; así como, para despejar inquietudes respecto al proceso que por falta de espacios participativos o interés, no se logran aclarar. Es así como, se estructuró el siguiente Plan de Manejo para ser desarrollado en este tipo de proyectos de conservación, y así evitar que solo la parte técnica tenga incidencia en la conservación de la flora epífita; fortaleciendo la gobernanza ambiental, al incluir la participación de la comunidad local.

Para ello se propone un trabajo integrador de los actores educativos (Universidades, Colegios, Centros de investigaciones), los actores institucionales (entidades públicas) y actores civiles/entidades privadas (comunidades locales, junta de acción comunal, sindicatos, ONG's ), para que se genere interés en los procesos de rescate de las epífitas y de otras problemáticas ambientales regionales y locales, donde todos tengan conocimiento de las situaciones y apoyen desde sus capacidades, saberes, equipamientos y experiencias. Para lograr la integralidad de los actores se deben definir las metas, responsabilidades de cada actor social, el tiempo de diseñoejecución- finalización de los procesos, lo cual con lleva a realizar una Planificación, seguimiento y evaluación de las mismas, acciones que permitan identificar falencias, fortalezas y cambios necesarios para cumplir a cabalidad lo estipulado en las metas. Todo esto abordándose desde una información transparente y clara, cumpliendo los requerimientos de las resoluciones establecidas por el gobierno y generándose espacios participativos para todos los actores (ver Fig. 4.).

El Plan de manejo fue presentado a los actores sociales de la vereda "El Páramo" el día 16 de octubre del 2020 en la Reserva "La Manuela", puntualmente en el epifitario conformado por la reubicación. El lugar del encuentro fue definido por el desconocimiento de algunos de la ubicación y del proceso que llevan las plantas, así que desde esta propuesta se deseó ampliar la información y promover la participación, especialmente de actores locales. El grupo focal estuvo conformado por 12 personas, las cuales representaron los siguientes actores: CORPONOR, Universidad de Pamplona, Empresa encargada de reubicación, Alcaldía de pamplonita, junta de acción comunal y representantes de agricultores y de la comunidad local.

Al darles a conocer el Plan de manejo propuesto hubo aceptación por parte de los líderes convocados. Se resalta por parte de los actores claves las siguientes consideraciones:

"Las relaciones permiten desarrollar proyectos, si no las hay no prosperan las cosas. Esa es una problemática a nivel nacional, no se vincula a solucionar los problemas regionales con la comunidad".

"Las universidades deben contribuir por el desarrollo regional y se ha perdido en la región, para restablecerlo o hacerlo, se empieza con estos tipos de encuentros. Con la corporación se interactúa un poco más".

"La mejor garantía que tiene los rescates es que se dé continuamente la interacción con la comunidad".

"Por ahora debemos empezar con los niños. Lamento que los niños no puedan estar por la pandemia acá, les queremos inculcar 
esto, son el futuro de mañana en la vereda y son los que a futuro van a disfrutar de este ecosistema. Nosotros los tenemos ahí en mente para que sean líderes en cuidar el agua, y estudien lo relacionado con la conservación".
"Se puede pensar en vincular la comunidad desde los proyectos de grado de la universidad, inventariando y participando en el conocimiento de los recursos vinculándolo a ellos, reconociendo sus saberes. La comunidad aporta mucha información de los sitios donde mejor prosperan las especies".

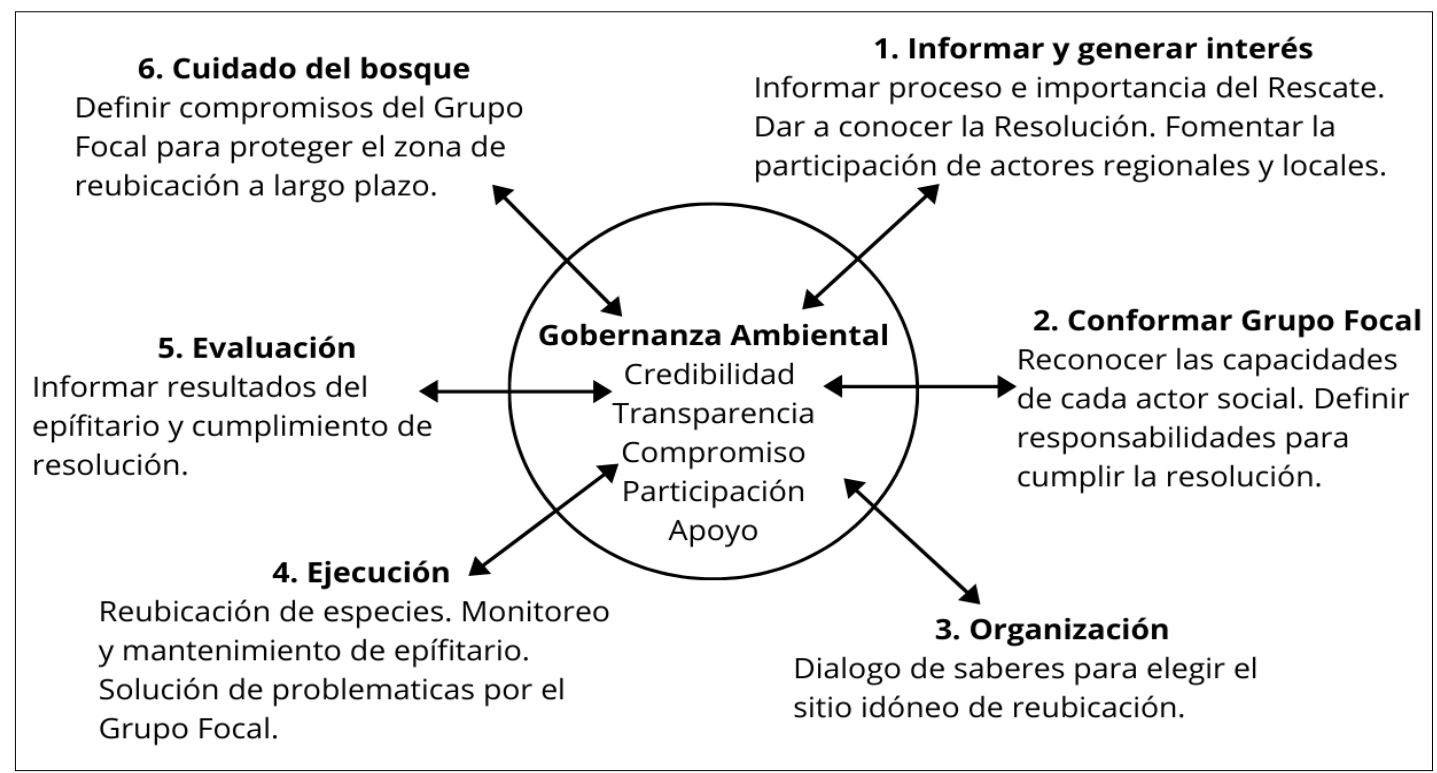

Figura 4. Plan de manejo para reubicación de flora epífita desde un enfoque de Gobernanza ambiental.

Una parte muy importante en este espacio participativo generado fue acercar a la comunidad local al proceso metodológico que se realiza para el traslado y reubicación de la flora epifita, esto con el fin de generar confiabilidad en el proceso de conservación. Se realizó un recorrido por los árboles hospederos escogidos para amarrar las epifítas, lo que permitió identificar formación de nuevas raíces que permiten una adhesión positiva sobre el nuevo forofito, formación de frutos y flores que nos indican presencia de polinizadores, y en general idoneidad del sitio para la proliferación de estas especies. Esta actividad sencilla fortaleció el interés por la comunidad principalmente y dejo las siguientes consideraciones:

"Esto lleva año y medio y nunca había venido a ver como lo hacen y como la orquídea pega en el nuevo árbol, y pues veo que si se puede".

"Es el primer proyecto donde sube más gente a mirar".

"Este proyecto me parece muy bueno, no dejan morir esas plantas".

"Como comunidad siempre estamos atentos a los llamados que hacen las empresas y hay veces que se buscan de diferentes maneras de acercarnos para buscar árboles para plantar".

Ante la baja interacción entre los actores directos e indirectos evidenciada en esta investigación, se dieron a conocer las siguientes propuestas de cambio dadas por los mismos líderes:
"Se debe promover la alianza nuevamente de la corporación y la Universidad de Pamplona, debemos buscar la academia. Debemos reactivar el convenio donde se incluya la Universidad en la participación en la evaluación y seguimiento que se dan para las especies vedadas".

"Se debe buscar mesas técnicas en el ANLA que tengan en cuentan las observaciones que hace la corporación, la academia, y la comunidad".

"Se destaca la importancia que la comunidad campesina de participar en los diferentes proyectos que llegan a la vereda, y participar hacer uso del derecho de veedores".

"Es necesaria la presencia de la Universidad de Pamplona en los proyectos de la vereda, como en asesorar las siembras de árboles, ubicar las zonas idóneas, saber cómo se compone su biodiversidad, entre todos queda mejor".

"Se debe aprovechar este tipo de espacios. Todo parte de la curiosidad, de saber de los avances de proyectos, compartir información, aportar datos útiles para el tema de conservación, y solicitar ayuda a los otros actores. Creo que eso fortalecerá la unidad de la comunidad con las entidades que aquí se representan".

"Se reconoce la falta de interacción de las entidades educativas superiores con las comunidades, eso falta realmente. Se me ocurre que por medio de la divulgación se puede promover más la participación, y así la gente la reconoce y ven la importancia 
que tiene. Se destaca que las comunidades tienen su conocimiento, saben para que sirven la plantas como se usan, donde pueden prosperar. Así que para este tipo de Proyectos las comunidades de las veredas son importantes, pueden participar en los sitios donde es mejor reubicar las plantas, saben bien en donde crecen, sus observaciones en el tiempo son valiosas. El vínculo debe fortalecerse".

\section{Discusión}

Para Zúñiga y Gumucio [28] "temas que antes eran considerados de exclusiva injerencia de científicos, técnicos y/o expertos, convocan y movilizan ahora a distintos actores (ciudadanía, empresarios, autoridades políticas) en distintos niveles: local, regional, nacional e internacional" (p.3), dejando manifiesto que la articulación entre actores permite construir gobernanza. Calvo, Hoyos y Amaya [4], definen la gobernanza como la participación de los actores en la toma de decisiones y en la solución para problemas colectivos, dado que el conjunto de actores comparte un mismo territorio y de forma corresponsable potencian su transformación social, productiva e institucional.

Se hace necesario considerar todos los actores en las estrategias locales aplicadas para la conservación; más allá de solo informar, se debe buscar la participación de los mismos para generar una mayor apropiación del territorio. Se ve en la Fig. 1, que la mayoría de los actores indirectos con incidencia local no son incluidos en este proceso, tan solo son informados; en estos actores se incluyen la comunidad, los agricultores de la zona y el ente educativo de la vereda. Esta situación coincide con lo mencionado por Rodríguez y Muñoz [22], donde resaltan que en Colombia es común encontrar proyectos con escasa información y divulgación hacia las comunidades de influencia, donde en el proceso de toma de decisiones no son incluidos.

La participación de los actores en los diferentes procesos que requieren toma de decisiones permite manifestar los intereses propios, sus expectativas e inquietudes comunes, facilita el consenso de un determinado tema que los implique directa o indirectamente; y los imaginarios y percepciones que estos tengan frente a la naturaleza, hacen aún más necesario su inclusión en los diferentes procesos, ya que se generan expresiones territoriales reflejado en posiciones [25]. Se genera credibilidad por parte de la comunidad cuando se conoce como se ejecutan los proyectos y su finalidad, la participación se da cuando se incentiva una real democracia y se promueve el interés de cada uno de ellos por la situación, motivando acciones que tendrán efectos a futuro; además, al ser miembros de una sociedad, todas las personas tienen el derecho y el deber de hacer parte en los procesos que afecten su entorno [22].

Andrade et al [1], resaltan la importancia de involucrar el conocimiento de las diferentes comunidades (indígenas, campesinas y negritudes), en las investigaciones formales, dando reconocimiento a estas experiencias y fortaleciendo la apropiación del territorio, ya que al dar participación a la sociedad civil en lo relacionado a la conservación de la biodiversidad e integrarlo al nivel local, regional y nacional, se estará logrando una gobernanza participativa. Una participación efectiva de la comunidad logra mostrar la realidad de los problemas e identificar nuevas alternativas de solución que serán apoyadas por ellos mismos, y que pueden ser más efectivas que las impuestas, siendo que se generan compromisos directos ante las entidades ambientales y estatales, como también con sus vecinos, familiares y conocidos, con aquellas personas con las que convive día a día, y esto genera mayor responsabilidad [15].

Lograr la sustentabilidad de la flora epifita y de más servicios ecosistémicos de la flora, será más complejo e ineficiente sin la participación de todos los actores. Una gestión ambiental participativa es la mejor alternativa posible para alcanzar efectos de sostenibilidad ambiental y de calidad de vida para todos a corto, mediano y largo plazo. Ya lo mencionaron Rodríguez y Muñoz [22] "cualquier tipo de exclusión en lo ambiental termina siendo de alto riesgo" (p.98).

Los Planes de manejo de gobernanza aportan organización territorial, la disciplina en su ejecución permite mantener presente las metas y cambios que cada actor debe aportar, ya que las diferentes miradas con las que se aborde un conflicto social y ambiental, impulsan la transdisciplinariedad y se contrapone a la especialización que maneja la modernidad, con esto se tiene imágenes simples y concretas de la situación real a que se debe solucionar [17]. Flores [7] destaca la valiosa inclusión de las juntas de acción comunal para la gobernanza, siendo representantes de la comunidad local que interactúan con más cercanía con la demás ciudadanía, ya sea rural o urbana.

Garnica [8] manifiesta que además de tener espacios participativos e incluir a la comunidad local, los actores gubernamentales y educativos deben apoyar las propuestas con su conocimiento técnico, integrar la investigación, invertir en los cambios y ampliar las estrategias para un impulsar un desarrollo sustentable. Desde los grupos de investigación, las instituciones educativas de nivel superior, desarrollan mejoras en el conocimiento, la tecnología e impulsan la innovación, siendo potenciales agentes de cambio social [21]; por ello su interacción con las comunidades locales es de vital importancia para impulsar cambios. 


\section{Conclusiones}

Se evidenció desconocimiento por algunos actores sobre la continuidad del proceso, importancia y funcionalidad del mismo, y participación de otras entidades; dejando entre ver baja gobernanza ambiental por parte de los actores sociales de la vereda El Páramo. Para mejorar este proceso de conservación se propone un Plan de Manejo basado en principios de gobernanza ambiental, donde se busca un trabajo integrado por parte de todos los actores, que conste de espacios participativos e informativos de avances, reconocimiento de saberes, percepciones, responsabilidades y definición de metas puntuales, donde se busca aumentar la efectividad en los procesos de conservación, promover apropiación del territorio y desarrollar credibilidad en los proyectos de reubicación de epífitas por parte de la comunidad local.

\section{Reconocimientos}

A los líderes de cada actor social que participaron de manera voluntaria y dispusieron de su tiempo para apoyar los resultados de esta investigación.

\section{Referencias}

[1] Andrade, G. I., Albán, F. C., Umaña, J. C., Nannetti, E. G., Toro, B. L., Mance, H., ... \& Lleras, G. R. Gobernabilidad, instituciones y medio ambiente en Colombia. Foro Nacional Ambiental. Bogotá. 568. pp. 2008

[2] Bonilla, J., \& Escobar, J. Grupos focales: una guía conceptual y metodológica. Cuadernos Hispanoamericanos de Psicología, 9(1) (2017): 51-67.

[3] Brenner, L. Desarrollo local participativo y buena gobernanza ambiental: ¿ una combinación viable? La experiencia de una Reserva de la Biosfera mexicana. Medio Ambient. Urban, 76 (1) (2012): 211-242. ISSN 1870-3925.

[4] Calvo, G., Hoyos, O., \& Amaya, M. Miradas cruzadas sobre el enfoque territorial de la responsabilidad social, la gobernanza y la sostenibilidad. Opera, 26 (2020): 3-14. https://doi.org/10.18601/16578651.n26.02

[5] CORPONOR. Propuesta de declaratoria de la Reserva Forestal Protectora Mejué, Municipios de Toledo, Chinácota, Herrán, Pamplonita, Norte de Santander. Cúcuta Ed. Corporación Autónoma Regional de la Frontera Nororiental. 2014. pp. 43

[6] CORPONOR. Planeación y Ordenamiento Territorial de la cuenca hidrica del Rio Pamplonita. Cúcuta. Ed. Corporación Autonoma de la Frontera Nororiental. 2009. pp. 38

[7] Flores, L. M. (2020). Gobernanza participativa, la experiencia de Barcelona. . Cuadernos de Vivienda y Urbanismo, 13: 1- 15. https://doi.org/10.11144/Javeriana.cvu13.gpeb

[8] Garnica, V. A. La gobernanza ambiental como enfoque para la cogestión adaptativa. Integra Educativa, 9(1) (2016): 159-168. ISSN 1997-4043.

[9] Gentry, A. H., \& Dodson, C. Contribution of non-trees to species richnes of tropical rain forest. Biotropica, 19. (1987): 149-156. https://doi: $10.2307 / 2388737$.

[10] Heredia, M., Bravo, C., Torres, B., \& Alemán, R. Innovación para el fortalecimiento de capacidades sobre sostenibilidad de los recursos naturales en poblaciones indígenas y mestizas - colonas: Reserva de la Bisofera Yusaní. Iberica de Sistemas e Tecnologias de Informacao, 16 (2020):103-116
[11] Indij, D., \& Schreider, M. P. Herramientas para contribuir a la gestión sustentable del agua en Latinoamérica. JRC Scientific and Technical Reports. 2015. pp.78

[12] Johansson, A. Mapeo y cuantificación de percepciones del cambio ambiental en el valle de Kilombero, Tanzania. Ambio, 49 (2) (2020): 557-568. https://doi.org/10.1007/s13280-019-01226-

[13] Krömer, T., \& Gradstein, S. R. Vascular epiphytes. Core Standardized Methods for Rapid Biological Field Assessment. Conservation International, Arlington, VA. Larsen, T.H. ed. 2016. pp. 26-32. ISBN: 978-1-934151-96-9

[14] Larrota, E. F. Propuesta metodológica para la caracterización de especies en veda de hábito epífito, terrestre y/o rupícola. Tesis Esp. Facultad de Ingeniería. Universidad Militar, Bogotá. 2018.

[15] Londoño-Toro, B., Torres-Villarreal, M. L., Lozano, M. A., Zuluaga, C., Arias Barreto, A. M., Pérez Muñoz, G., ... \& Cortés Monroy, T. 20 años de la Ley de Acciones Popular en Colombia: balance y desafíos 1998-2018. Grupo de Investigación en Derechos Humanos. Facultad de Jurisprudencia. Bogotá, Universidad del Rosario. 2019.

[16] Malambo, N. Propuesta de Gestión ambiental participativa y educativa para la participación comunitaria del proceso de restauración ecológica del parque forestal Embalse del Neusa. Tesis MSc, Facultad de Estudios ambientales y Rurales. Bogotá, Universidad Potificia Javeriana. 2017.

[17] Martínez, G. R., Caballero, J. M., Solís, P. C., \& Vargas, L. G. Introducción a los estudios organizacionales en el análisis de problemas sociales en América Latina. El Análisis Organizacional en el Desarrollo Local. Centro de Investigación y Desarrollo, Ecuador (CIDE). 2020. pp.230-247. ISBN: 9789942-802-32-3

[18] Naranjo Otálvaro, M. J., Rodríguez-Lora, V., Álvarez Arboleda, B., Silva Arroyave, S. M., Medina Restrepo, C. A., Mejía Alzate, M. L., ... \& Restrepo Ayala, C. E. El Análisis Organizacional en el Desarrollo Local. Centro de Investigación y Desarrollo, Ecuador (CIDE). 2020. pp. 221 229. ISBN: 978-9942-802-32-3

[19] OIMT \& UICN. Directrices OIMT/UICN para la conservación y utilización sostenible de la biodiversidad en los bosques tropicales productores de madera. Serie de políticas forestales OIMT 17(2009):1- 124

[20] Pérez, V. W. Modelo de gobernanza para la participación ciudadana en el Gobierno Regional, Amazonas. Tesis Doctorado, Facultad de Derecho y Humanidades. Perú, Universidad Cesar Vallejo. 2020

[21] Rodríguez-Lora, V. Los grupos de investigación vistos como organizaciones que aprenden. En: Analisis organizacional del Desarrollo Local. Guayaquil, Ecuador: Editorial Centro de Investigación y Desarrollo Ecuador (CIDE). 2020. pp 380-396. ISBN: 978-9942-802-32-3.

[22] Rodriguez, Gloria-Amparo \& Munoz Avila, Lina. La participación en la Gestión Ambiental: Un reto para el nuevo milenio. Facultad de Jurisprudencia. Bogotá, Universidad del Rosario, 2009. pp. 192. http://dx.doi.org/10.2139/ssrn.2592982

[23] Rubio, J. P. Evaluación de la efectividad de la normatividad ambiental legal vigente para la preservación de la diversdiad vegetal epífita. Tesis Esp. Facultad de Ingeniería. Bogotá. Universidad Militar Nueva Granada. 2015.

[24] Seid, G. Procedimientos para el análisis cualitativo de entrevistas. Una propuesta didáctica. V Encuentro Latinoamericano de Metodología de las Ciencias Sociales 16 al 18 de noviembre de 2016 Mendoza, Argentina. Métodos, metodologías y nuevas epistemologías en las ciencias sociales: desafíos para el conocimiento profundo de Nuestra América. Universidad Nacional de La Plata. Facultad de Humanidades y Ciencias de la Educación. Centro Interdisciplinario de Metodología de las Ciencias Sociales. 2016.

[25] Silva, B., \& Hernández, Y. Participación campesina en el ordenamiento ambiental territorial en la zona de reserva campesina. El caso de Cabrera Cundinamarca $(2000$ - 2017). Tesis pregrado, Facultad de Medio Ambiente y Recursos 
Naturales. Bogotá, Universidad Distrital Francisco José de Caldas. 2017.

[26] Trujillo, J. Juicio valorativo del proceso contractual de las vias4G en Colombia. Tesis MSc, Facultad de Ciencias Políticas y Relaciones Internacionales. Calí, Universidad Pontificia Javeriana. 2019.

[27] Varguillas, C. El uso de atlas.Ti y la creatividad del investigador en el análisis cualitativo de contenido upel. Laurus.12 (2006): 7387. ISSN: $1315-883 X$.

[28] Zúñiga, M. P., \& Gumucio, C. P. Funcionarios públicos y evaluación ambiental en Chile: tensiones en la construcción de una gobernanza ambiental democrática. Revista Mexicana de Ciencias Políticas y Sociales 65 (2020): 379-393. http://dx.doi.org/10.22201/fcpys.2448492xe.2020.239.67896 